\title{
Asistencia ventilatoria no invasiva domiciliaria en niños: impacto inicial de un programa nacional en Chile
}

\author{
FRANCISCO PRADO A. ${ }^{1}$, PAMELA SALINAS F. ${ }^{2}$
}

1. Médico Neumólogo Pediatra, Programa Asistencia Ventilatoria No Invasiva, Unidad de Salud Respiratoria, División de Atención Primaria en Salud, Ministerio de Salud de Chile 2006-2009. Departamento de Pediatría Campus Centro, Facultad de Medicina, Universidad de Chile.

2. Enfermera, Programa Asistencia Ventilatoria No Invasiva, Unidad de Salud Respiratoria, División de Atención Primaria en Salud, Ministerio de Salud de Chile 2006-2009.

\begin{abstract}
At-home non-invasive ventilatory assistance for children: initial impact of a national program in Chile
\end{abstract}

Introduction: Prolonged mechanical ventilation may be provided to children with chronic ventilatory failure as non-invasive ventilatory assistance at home (NIVA). Objective: To describe clinical characteristics, evolution outcomes and Health Related Quality of Life (HRQOL) of pediatric patients admitted into the Chilean NIVA program. Patients and Methods: Medical files of patients included in the program, were reviewed during a period of 24 months (2006-2008), using follow-up protocols. Results: There were a total of 177 children, with an average age of $9.7 \pm 4.7$ years. Fourteen patients died of non-related causes. Diagnoses were: neuromuscular disease (NMD) 64\%, myelomeningocele 6\%, obstructive sleep apnea syndrome 6\%, lung disease $20 \%$ and miscellaneous $4 \%$. Compared to the previous year, hospitalization decreased from 60 to $21 \%$ $(\mathrm{p}<0.00001)$ and from 1.3 hospitalizations/patient/year to $0.3(\mathrm{p}<0.00001)$. HRQOL improved significantly, AUQUEI $17.2 \pm 5.6(\mathrm{p}<0.05)$, IRS $26.4 \pm 8.2$ points $(\mathrm{p}<0.05)$ in 82 patients. In 21 patients with NMD an average increase of $27 \mathrm{cmH}_{2} \mathrm{O}$ on muscular inspiratory pressure $(\mathrm{p}<0.05)$ after respiratory training was observed. Conclusions: The NIVA program reduced hospitalizations and improved HRQOL. In a group of patients the muscle strength increased with stable respiratory function.

(Key words: Non invasive ventilation, Children, Home Care, National Program).

Rev Chil Pediatr 2011; 82 (4): 289-299

\section{RESUMEN}

Introducción: La asistencia ventilatoria no invasiva domiciliaria (AVNI) es una modalidad de ventilación mecánica prolongada que puede ser proporcionada a niños con insuficiencia ventilatoria crónica. Objetivo:

Trabajo recibido el 24 de febrero de 2011, devuelto para corregir el 07 de abril de 2011, segunda versión el 12 de abril de 2011, tercera versión el 09 de mayo de 2011, aceptado para publicación el 16 de mayo de 2011.

Correspondencia a:

Dr. Francisco Prado A.

E-mail: panchoprado2004@gmail.com 
Describir las características clínicas, resultados en su evolución y calidad de vida relacionada a salud (CVRS) en pacientes ingresados a un programa nacional de AVNI. Pacientes y Métodos: Se revisaron los registros de pacientes del programa durante 24 meses (2006-2008). Se utilizó un protocolo de seguimiento previamente definido. Resultados: 177 niños, edad promedio 9,7 \pm 4,7 años. Catorce fallecieron por causas no relacionadas a la AVNI. Diagnósticos: enfermedad neuromuscular (ENM) 64\%, mielomeningocele operado 6\%, síndrome apnea obstructiva del sueño 6\%, enfermedad del parénquima pulmonar 20\% y miscelánea 4\%. Respecto al año previo al ingreso las hospitalizaciones disminuyeron de 60 a 21\% (p < 0,00001) y de 1,3 hospitalizaciones/ paciente/año a 0,3 ( $\mathrm{p}<0,00001)$. En 82 pacientes la CVRS mejoró, AUQUEI 17,2 \pm 5,6 puntos ( $<<0,05)$, IRS $26,4 \pm 8,2$ puntos $(\mathrm{p}<0,05)$. En 21 pacientes con ENM y entrenamiento respiratorio hubo mejoría de la Pimax $\left(27 \mathrm{cmH}_{2} \mathrm{O} ; \mathrm{p}<0,05\right)$ y no hubo disminución en la CVF. Conclusiones: AVNI disminuyó las hospitalizaciones y mejoró la CVRS de los pacientes ingresados al programa nacional. En un grupo de pacientes mejoró la fuerza muscular con estabilización de la función respiratoria.

(Palabras clave: Ventilación no invasiva, niños, atención domiciliaria, programa nacional).

Rev Chil Pediatr 2011; 82 (4): 289-299

\section{Introducción}

La asistencia ventilatoria no invasiva domiciliaria (AVNI) es una modalidad de ventilación mecánica efectiva en el manejo de pacientes con apneas obstructivas del sueño por alteración de la vía aérea superior, y pacientes con compromiso primario (enfermedades neuromusculares) o secundario de la bomba respiratoria (fibrosis quística, enfermedad pulmonar obstructiva crónica), tanto en exacerbaciones respiratorias agudas como en pacientes con insuficiencia respiratoria crónica. En estas dos últimas condiciones y escenarios clínicos, agudo y crónico, con hipoventilación e hipercapnia ${ }^{1-4}$.

Cuando existe compromiso de la bomba respiratoria la AVNI mejora la ventilación alveolar y el intercambio gaseoso, estabiliza la declinación de la función pulmonar, mejora los trastornos respiratorios del sueño (TRS) y disminuye las exacerbaciones. Optimizando los costos sanitarios, la calidad de vida relacionada con salud (CVRS) y mejorando la sobrevida $^{5-7}$.

La heterogeneidad y complejidad de los diagnósticos y enfermedades de base han determinado que la experiencia pediátrica domiciliaria sea restringida. Sin embargo, diversas estrategias de ventilación mecánica prolongada (VMP) y necesidades tecnológicas complementarias como tos asistida, tienen un rol creciente $^{1}$. Las publicaciones que han revisado su impacto en la historia natural de la enfer- medad, especialmente en las enfermedades neuromusculares (ENM) y cifoescoliosis, recomiendan su inicio precoz al pesquisar hipoventilación nocturna, condición que se adelanta a los trastornos de función pulmonar y gases sanguíneos en vigiliaa ${ }^{2,5,8}$. La evaluación periódica, que incluye estudios de los TRS, hipoventilación nocturna, función pulmonar y fuerza de la musculatura respiratoria y no respiratoria, permite decidir el inicio precoz de la AVNI ${ }^{5,7,8,10-12}$.

En Chile existe experiencia con un número reducido de pacientes ${ }^{13-15}$ y otros reportes en Latinoamérica son escasos ${ }^{9}$.

En el año $2006^{16}$ se inició el Programa Nacional en la Atención Primaria en Salud para abordar en forma sistemática las necesidades de AVNI domiciliaria en niños y adolescentes con hipoventilación nocturna derivada de diversas patologías de la bomba respiratoria, vía área o parénquima pulmonar, integrando distintos niveles de atención en la red asistencial, considerando el hogar y la familia como eje central en la entrega de la AVNI. Su objetivo fue mejorar la expectativa y CVRS, junto con disminuir los gastos asociados a VMP, en pacientes cuyo único escenario posible era estar hospitalizados en unidades de paciente crítico.

El objetivo de esta comunicación es describir las características clínicas, tipo de asistencia ventilatoria, junto con los resultados del seguimiento y evolución en un grupo de niños con VMP, incorporados al Programa Chileno de AVNI en domicilio en su etapa inicial. 


\section{Sujetos y Método}

Estudio descriptivo de los registros electrónicos (fichas clínicas) de una serie de 177 pacientes incorporados a un programa nacional de AVNI en domicilio, beneficiarios del Fondo Nacional de Salud (FONASA), con acciones de intervención y seguimiento protocolizado por un período de 24 meses (agosto 2006-agosto 2008). Los criterios de selección, inclusión y exclusión al Programa AVNI en domicilio se presentan en la tabla 1, para la inclusión de pacientes no se considero como obligatoriedad exámenes que objetivaran la hipoventilación nocturna como polisomnografía o capnografía en aquellos pacientes que presentaban signos y síntomas sugerentes con deterioro clínico o funcional diurno evidente, y además por la baja disponibilidad de estos exámenes en los centros hospitalarios a nivel nacional por limitaciones de recursos. Todos los pacientes ingresados al programa recibieron prestaciones en sus domicilios por profesionales de la salud contratados en la atención primaria de salud o por medio de servicios externalizados a través de licitación pública centralizada. Estos profesionales, kinesiólogo, enfermera y médico, actuaban como facilitadores del proceso de autocuidado, entregando educación continua en relación al manejo de la enfermedad, equipamientos, acciones frente a descompensaciones y actividades de promoción en salud, de acuerdo a los requerimientos del grupo familiar.

Entre las áreas complementarias abordadas por el Programa AVNI, se creó un protocolo de entrenamiento de la musculatura respiratoria (EMR), un protocolo de evaluación de CVRS $\mathrm{y}$ un protocolo de contingencias en respuesta a

Tabla 1. Criterios de Inclusión y Exclusión para el Programa AVNI

\begin{tabular}{|c|c|c|}
\hline Criterios de selección & Inclusión & Exclusión \\
\hline $\begin{array}{l}\text { Condición clínica estable, sin cambios } \\
\text { importantes de los parámetros de AVNI en } \\
\text { las últimas tres semanas }\end{array}$ & $\begin{array}{l}\text { Pacientes con insuficiencia respiratoria } \\
\text { crónica y síndromes de hipoventilación } \\
\text { nocturna secundarios a patología que } \\
\text { cumplan los siguientes criterios: } \\
\text { - Saturometría nocturna continua anor- } \\
\text { mal ( } \mathrm{SpO}_{2}<90 \%>10 \% \text { del tiempo de } \\
\text { sueño) } \\
\text { - } \mathrm{CVF}<50 \% \text { valor predicho, Pimax }<40 \\
\mathrm{~cm} \mathrm{H} \mathrm{H}_{2} \mathrm{O}, \mathrm{PFT} \text { tos bajo } 150 \mathrm{l} / \mathrm{m} \\
\text { - } \mathrm{GSA}: \mathrm{PaCO}_{2}>50 \mathrm{mmHg} \text {, EB }>4 \mathrm{mEq} / \mathrm{l}\end{array}$ & $\begin{array}{l}\text { - Enfermedad neuromuscular de progre- } \\
\text { sión rápida } \\
\text { - Parálisis cerebral }\end{array}$ \\
\hline $\begin{array}{l}\text { Necesidad de soporte ventilatorio noctur- } \\
\text { no por tiempos de permanencia no mayor } \\
\text { a } 10 \text { hrs }\end{array}$ & $\begin{array}{l}\text { Pacientes con enfermedad neuromuscular } \\
\text { de progresión lenta o estacionaria }\end{array}$ & $\begin{array}{l}\text { - Falta total de tolerancia a interfase } \\
\text { - Trastorno de deglución con ausencia de } \\
\text { protección glótica }\end{array}$ \\
\hline $\begin{array}{l}\text { Hipercapnea sin acidosis respiratoria. Re- } \\
\text { querimiento de oxígeno }<\text { de } 2 \mathrm{l} / \mathrm{m} \text { para } \\
\text { mantener } \mathrm{SpO}_{2}>93 \%\end{array}$ & $\begin{array}{l}\text { Pacientes con compromiso primario o } \\
\text { secundario del comando ventilatorio y } \\
\text { síndrome de apneas obstructivas del sueño } \\
\text { (SAOS) con: } \\
\text { - } \mathrm{SpO}_{2} \text { frecuente }<90 \% \text { en registro con- } \\
\text { tinuo por al menos } 8 \mathrm{~h} \text { de observación } \\
\text { - Índice de apneas } / \text { hipopneas }>5 / \mathrm{hr}^{32} \text {, } \\
\mathrm{CO}_{2} \text { transcutáneo }>50 \text { mmHg por más } \\
50 \% \text { tiempo registro nocturno }{ }^{2,33}\end{array}$ & $\begin{array}{l}\text { Necesidad de soporte ventilatorio por más } \\
\text { de } 10 \text { hrs o ausencia de autonomía respi- } \\
\text { ratoria fuera de soporte de presión positiva }\end{array}$ \\
\hline IPAP/EPAP no superior a $20 / 8 \mathrm{cmH}_{2} \mathrm{O}$ & & Menor de 6 meses (relativo) \\
\hline $\begin{array}{l}\text { Situación socioeconómica con instalacio- } \\
\text { nes y servicios básicos apropiados. Nivel } \\
\text { de instrucción que permita cumplimiento } \\
\text { de indicaciones }\end{array}$ & & $\begin{array}{l}\text { No cumplir con los criterios de evaluación } \\
\text { social referidos en los criterios de inclusión }\end{array}$ \\
\hline $\begin{array}{l}\text { Cuidadores comprometidos con el segui- } \\
\text { miento y los controles }\end{array}$ & & $\begin{array}{l}\text { Imposibilidad de mantener controles de } \\
\text { seguimiento al alta }\end{array}$ \\
\hline
\end{tabular}

AVNI: Asistencia Ventilatoria No Invasiva, $\mathrm{SpO}_{2}$ : Saturación de oxígeno, CVF: Capacidad vital forzada, Pimax: Presión inspiratoria máxima, PFT: Pico flujo tos, GSA: Gases sanguíneos arteriales, EB: Exceso de base. 
las emergencias por perdida de la estabilidad clínica.

Con objeto de conocer la complejidad de los pacientes beneficiarios del Programa AVNI y definir las demandas para su atención en domicilio se utilizó un instrumento para la categorización de usuarios por dependencia y riesgo de los cuidados (CUDYR), identificando su complejidad $^{17}$.

\section{Protocolo de entrenamiento de la musculatura respiratoria (EMR)}

El protocolo de EMR utilizó como carga de trabajo el $30 \%$ de la presión inspiratoria máxima (Pimax) generada a través de válvulas de umbral regulable (Treshold ${ }^{\circledR}$ IMT, Philips Respironics), cuya resistencia es independiente del flujo, y permiten fijar la carga de entrenamiento. El entrenamiento se desarrolló en intervalos incrementales de 3, 5, 10 y 15 minutos, con 2 minutos máximo de descanso interseries durante 5 días a la semana, progresando según tolerancia. Se realizó en niños mayores de seis años con habilidades neurocognitivas y condición clínica que permitían su colaboración con Pimax menor de $80 \mathrm{~cm}$ de $\mathrm{H}_{2} \mathrm{O}^{18}$.

En 26 pacientes con ENM, al inicio del EMR, se evaluó la función pulmonar (espirometría) y se correlacionó con la Pimax utilizando r de Pearson. La Pimax se obtuvo según la técnica descrita por Black y Hyatt para el registro de las presiones máximas generadas en la boca ${ }^{19}$ y las espirometrías según las recomendaciones de la Sociedad Americana del Tórax (ATS) ${ }^{20}$. Los resultados obtenidos se expresaron como porcentaje del valor predicho utilizando los valores de referencia de Szeinberg $^{21}$ y Knudson ${ }^{22}$, respectivamente.

En aquellos pacientes que siguieron rutinariamente el EMR se midió Pimáx al inicio y luego cada 3 meses por un período de 18 meses y la capacidad vital forzada (CVF) al inicio y a los 12 meses de seguimiento. Los valores de Pimax se expresaron como valor absoluto en $\mathrm{cmH}_{2} \mathrm{O}$.

\section{Protocolo de evaluación de la calidad de vida relacionada con salud}

Este protocolo, incluyó la utilización de distintos instrumentos genéricos y específicos:
Autoquestionnaire Qualité de Vie-Enfant-Imagé-AUQUEI, Cuestionario de Insuficiencia Respiratoria Severa-IRS, Escala de Impacto Familiar-IFS, Cuestionario de Salud de Goldberg y Apgar familiar ${ }^{23}$, que permitieran conocer diversos dimensiones de la CVRS al ingreso del Programa, a los 6 meses y al año. En este reporte se presenta la evaluación de 82 pacientes de la Región Metropolitana en un año de seguimiento.

\section{Protocolo de plan de contingencias frente a emergencias}

La evaluación de los eventos que constituyen un quiebre del basal clínico de los enfermos se abordó con un Protocolo de Plan de Contingencias frente a emergencias, falla de equipos u otras situaciones que provocaron alarma en el niño y/o familia (figura 1). En estos casos los usuarios del Programa recurrieron a una línea telefónica (Salud Responde del Ministerio de Salud, 6003607777), que entrega información, orientación y asistencia telefónica las 24 horas del día, a través de un flujograma de acciones y respuestas categorizadas por un profesional de la salud según lo que el cuidador refiera, contrastándolas con la información obtenida en la ficha electrónica. Esta línea telefónica, operada por profesionales de la salud, apoya el cuidado en el domicilio de los pacientes, permitiendo la resolución de las contingencias en el nivel de la red asistencial que sea más eficiente, ya sea contactando a los profesionales a cargo del paciente o al servicio de atención médica de urgencia (SAMU), en caso de ser necesario la resolución inmediata. Se analizaron los registros electrónicos de 159 pacientes ingresados entre el año 2006 y 2007, describiendo el número de eventos, su nivel de complejidad y resolución, además se comparó el número de hospitalizaciones en el año previo al ingreso del Programa y las hospitalizaciones registradas durante un año de prestaciones.

\section{Análisis estadístico}

Se realizó un análisis descriptivo mediante distribuciones de frecuencias para las variables cualitativas y promedio \pm desviación estándar para las variables cuantitativas. Se efectuaron 


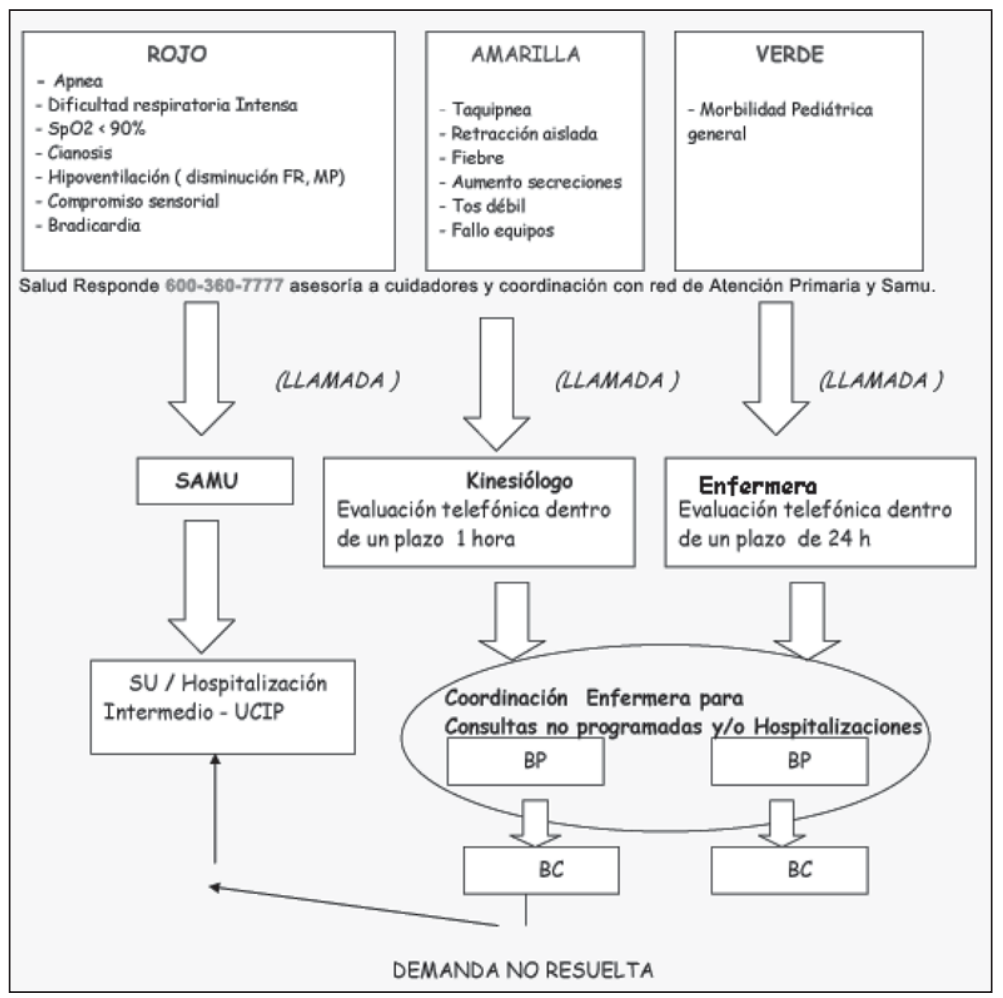

Figura 1. Protocolo de plan de contingencias frente a emergencias, falla de equipos $u$ otras situaciones que provocan alarma en el niño y/o familia. $\mathrm{SpO}_{2}$ : Saturación de oxígeno. MP: Murmullo pulmonar. SAMU: Servicio de atención médica de urgencia. BP: Broncopulmonar. BC: Broncopulmonar coordinador. SU: Servicio de urgencia. UCIP: Unidad de cuidados intensivos pediátrico. pruebas de normalidad Kolmogorov-Smirnov o Shapiro-Wilks, para definir tipos de análisis paramétricos (T de Student) o no paramétricos (prueba de rangos con signos de Wilcoxon). Se consideraron significativos los valores de $p<0,05$. Se utilizó el programa estadístico Statistical Package for the Social Sciences (SPSS) versión 15.0 (USA, 2006) para Windows ${ }^{\circledR}$.

\section{Resultados}

Las características clínicas de los pacientes ingresados al Programa de AVNI, diferenciadas según diagnóstico, se describen en la tabla 2. La edad promedio de los pacientes fue de 9,7 \pm 4,7 años (rango entre 5 meses y 20 años). La distribución según rangos de edad fue: $<$ de 6 años 28,5\%; 6 a 13 años 37\% y > 13 años $34,5 \%$. Setenta y un pacientes fueron hombres (61,7\%).

Los diagnósticos fueron: ENM/cifoescoliosis en 114 pacientes (64\%), enfermedad del parénquima pulmonar con insuficiencia respi- ratoria crónica hipercápnica (fibrosis quística, bronquiolitis obliterante post viral, displasia broncopulmonar y misceláneas) en 35 (20\%), mielomeningocele operado en 11 (6\%), apnea obstructiva del sueño en 10 (6\%) y misceláneas en 7 (4\%).

Al finalizar los 24 meses de revisión, 154 de los 177 pacientes ingresados se encontraban activos. Nueve pacientes $(5,1 \%)$ fueron dados de alta y catorce pacientes fallecieron (8\%) por causas no asociadas al uso de AVNI (evolución natural de la enfermedad y un paciente por sepsis abdominal secundaria a desplazamiento intraperitoneal de una sonda de gastrostomía). La AVNI fue entregada con generador de flujo y presión bi-nivelada (BiPAP) en modalidad espontánea o espontáneo/programado con mascarilla nasal preferentemente. Cincuenta y dos pacientes $(29,4 \%)$ con enfermedad del parénquima pulmonar requirieron además oxígenoterapia prolongada una vez iniciada la AVNI para mantener $\mathrm{SpO}_{2}$ mayor a 90\%. Cincuenta y cuatro (31\%) recibieron EMR.

La CUDYR fue realizada en 82 beneficia- 
PRADO F. y cols.

Tabla 2. Caracterización de los beneficiarios del Programa AVNI, según diagnóstico

\begin{tabular}{|c|c|c|c|c|c|}
\hline & $\begin{array}{l}\text { Enf. neuro- } \\
\text { muscular } \\
(n=114)\end{array}$ & $\begin{array}{l}\text { Enf. del } \\
\text { parénquima } \\
\text { pulmonar } \\
(\mathbf{n}=\mathbf{3 5})\end{array}$ & $\begin{array}{l}\text { SAOS } \\
(n=10)\end{array}$ & $\begin{array}{c}\text { MMC } \\
\text { operado } \\
(n=11)\end{array}$ & $\begin{array}{l}\text { Miscelánea } \\
\qquad(n=7)\end{array}$ \\
\hline $\begin{array}{l}\text { Estado a la fecha del reporte } \\
\text { Activo } \\
\text { Recuperado } \\
\text { Fallecido }\end{array}$ & $\begin{aligned} 101 & (88,5 \%) \\
4 & (3,5 \%) \\
9 & (8 \%)\end{aligned}$ & $\begin{aligned} 30 & (85,7 \%) \\
3 & (8,5 \%) \\
2 & (5,8)\end{aligned}$ & $\begin{array}{l}7(70 \%) \\
2(20 \%) \\
1(10 \%)\end{array}$ & $\begin{array}{l}10(91 \%) \\
1(9 \%)\end{array}$ & $\begin{array}{l}6(85,7 \%) \\
1(14,3 \%)\end{array}$ \\
\hline Edad & $9,5 \pm 4,7$ & $8,1 \pm 5,3$ & $8,3 \pm 4$ & $11,4 \pm 4$ & $5,1 \pm 2,7$ \\
\hline $\begin{array}{l}\text { Sexo } \\
\text { Femenino } \\
\text { Masculino }\end{array}$ & $\begin{array}{l}43(37,7 \%) \\
71(62,3 \%)\end{array}$ & $\begin{array}{ll}14 & (40 \%) \\
21 & (60 \%)\end{array}$ & $\begin{array}{l}5(50 \%) \\
5(50 \%)\end{array}$ & $\begin{array}{l}7(63,6 \%) \\
4(36,3 \%)\end{array}$ & $\begin{array}{c}3(42,8 \%) \\
4(57,2 \%)\end{array}$ \\
\hline $\begin{array}{l}\text { Terapia ventilatoria } \\
\text { Generador de flujo de presión binivelada } \\
\text { Modalidad } \mathrm{S} \\
\text { IPAP }\left(\mathrm{cmH}_{2} \mathrm{O}\right) \\
\text { EPAP }\left(\mathrm{cmH}_{2} \mathrm{O}\right) \\
\text { Modalidad } \mathrm{S} / \mathrm{T} \\
\text { IPAP }\left(\mathrm{cmH}_{2} \mathrm{O}\right) \\
\text { EPAP }\left(\mathrm{cmH}_{2} \mathrm{O}\right) \\
\text { Frecuencia respiratoria } \\
\text { Generador de flujo de presión continua } \\
\text { en la vía aérea } \\
\text { CPAP }\left(\mathrm{cmH}_{2} \mathrm{O}\right)\end{array}$ & $\begin{aligned} 27 & (23,7 \%) \\
10 & \pm 3 \\
5 & \pm 2 \\
87 & (76,3 \%) \\
14 & \pm 3 \\
7 & \pm 2 \\
14 & \pm 3\end{aligned}$ & $\begin{array}{c}17(48,5 \%) \\
11 \pm 2 \\
6 \pm 2 \\
17(48,5 \%) \\
12 \pm 2 \\
6 \pm 1 \\
10 \pm 4 \\
1(2,9 \%) \\
6\end{array}$ & $\begin{array}{c}4(40 \%) \\
8 \pm 1 \\
4 \pm 1\end{array}$ & $\begin{array}{l}5(45,5 \%) \\
10 \pm 2 \\
4 \pm 2 \\
6(54,5 \%) \\
11 \pm 2 \\
5 \pm 2 \\
10 \pm 1\end{array}$ & $\begin{array}{l}3(42,8 \%) \\
11 \pm 1 \\
5 \pm 1 \\
4(57,2 \%) \\
12 \pm 2 \\
5 \pm 1 \\
12 \pm 3\end{array}$ \\
\hline $\begin{array}{l}\text { Interfase } \\
\text { Nasal } \\
\text { Nasobucal } \\
\text { Complicaciones locales (úlceras por } \\
\text { presión en sitios de apoyo) }\end{array}$ & $\begin{array}{c}111(97,3 \%) \\
3(2,6 \%) \\
3\end{array}$ & $\begin{array}{c}35(100 \%) \\
0 \\
1\end{array}$ & $\begin{array}{c}9(90 \%) \\
1(10 \%) \\
0\end{array}$ & $\begin{array}{c}11(100 \%) \\
0 \\
0\end{array}$ & $\begin{array}{c}6(85,7 \%) \\
1(14,3 \%) \\
0\end{array}$ \\
\hline $\begin{array}{l}\text { Otras prestaciones } \\
\text { Oxígenoterapia } \\
\text { Entrenamiento muscular específico }\end{array}$ & $\begin{array}{l}19(16,6 \%) \\
31(27,2 \%)\end{array}$ & $\begin{array}{l}26(74,3 \%) \\
14(40 \%)\end{array}$ & $\begin{array}{l}2(20 \%) \\
1(10 \%)\end{array}$ & $\begin{array}{l}3(27,3 \%) \\
6(54,5 \%)\end{array}$ & $\begin{array}{l}2(28,5 \%) \\
2(28,5 \%)\end{array}$ \\
\hline $\begin{array}{l}\text { Morbilidad } \\
\text { Hospitalizaciones año previo a AVNI } \\
\text { Hospitalización durante un año en AVNI }\end{array}$ & $\begin{array}{l}1,3 \pm 1 \\
0,3 \pm 0,5^{*}\end{array}$ & $\begin{array}{l}1,7 \pm 1,4 \\
0,4 \pm 0,3^{*}\end{array}$ & $\begin{array}{l}1,1 \pm 1,1 \\
0,2 \pm 0,4^{*}\end{array}$ & $\begin{array}{l}1,6 \pm 0,7 \\
0,3 \pm 0,5^{*}\end{array}$ & $\begin{array}{l}0,8 \pm 0,8 \\
0,3 \pm 0,6\end{array}$ \\
\hline $\begin{array}{l}\text { Parámetros funcionales } \\
\text { Número de pacientes } \\
\text { Pimax }\left(\mathrm{cm} \mathrm{H}_{2} \mathrm{O}\right) \text { inicio Programa } \\
\text { Pimax }\left(\mathrm{cm} \mathrm{H}_{2} \mathrm{O}\right) \text { a los } 12 \text { meses } \\
\text { \% Predicho CVF inicio Programa } \\
\% \text { Predicho CVF a los } 12 \text { meses } \\
\text { \% Predicho VEF inicio Programa } \\
\text { \% Predicho } \mathrm{VEF}_{1} \text { a los } 12 \text { meses }\end{array}$ & $\begin{array}{l}21 \\
67 \pm 22 \\
94 \pm 24^{* *} \\
61,1 \pm 26,6 \\
63,2 \pm 31,8 \\
59,7 \pm 25,5 \\
62 \pm 31\end{array}$ & & & & \\
\hline $\begin{array}{l}\text { Calidad de vida relacionada con salud } \\
\text { Número de pacientes } \\
\text { AUQUEl inicio } \\
\text { AUQUEl a los } 12 \text { meses } \\
\text { IRS inicio } \\
\text { IRS a los } 12 \text { meses } \\
\text { IFS inicio } \\
\text { IFS a los } 12 \text { meses }\end{array}$ & $\begin{array}{l}61 \\
50,3 \pm 12,3 \\
69,7 \pm 7,8^{* *} \\
49 \pm 21,1 \\
87 \pm 10,6^{* *} \\
43,2 \pm 17 \\
55 \pm 12,3\end{array}$ & $\begin{array}{l}12 \\
53,4 \pm 9,5 \\
73,9 \pm 4,2^{* *} \\
54 \pm 17,7 \\
89,1 \pm 6^{* *} \\
53 \pm 8,6 \\
61,4 \pm 4,5\end{array}$ & $\begin{array}{l}2 \\
58,1 \pm 7,6 \\
72,7 \pm 3,1 \\
62 \pm 24,6 \\
85,3 \pm 8,4 \\
51,5 \pm 3,9 \\
59,8 \pm 10\end{array}$ & $\begin{array}{l}5 \\
56,4 \pm 5,4 \\
71,2 \pm 2,7 \\
52,9 \pm 25,4 \\
82,7 \pm 9,2 \\
47,5 \pm 6,6 \\
61,2 \pm 7,8\end{array}$ & $\begin{array}{l}2 \\
55,7 \pm 4,7 \\
74,5 \pm 5 \\
65,1 \pm 10,3 \\
84,8 \pm 4,6 \\
49,1 \pm 7,2 \\
62 \pm 3,4\end{array}$ \\
\hline
\end{tabular}

SAOS: Síndrome de apneas obstructiva del sueño, MMC: Mielomeningocele, IPAP: Presión positiva inspiratoria en la vía aérea, EPAP: Presión positiva espiratoria en la vía aérea, CPAP: Presión positiva continua en la vía aérea, $\mathrm{cmH}_{2} \mathrm{O}$ : centímetros de agua, AVNI: Asistencia ventilatoria no invasiva, Pimax: Presión inspiratoria máxima, CVF: Capacidad vital forzada, VEF 1 : Volumen espiratorio forzado al primer segundo, AUQUEI: Cuestionario autoaplicado de calidad de vida para niños con imágenes, IRS: Cuestionario insuficiencia respiratoria severa; IFS: Escala de impacto familiar.

Los valores de la tabla, pueden ser promedio \pm DS ó Número (\%). ${ }^{*} p<0,0001,{ }^{* *} p<0,05$. Prueba T de Student para variables CVRS, Pimax y Prueba de rango con signos de Wilcoxon para variable hospitalizaciones. 
rios de la Región Metropolitana. La categorización por dependencia demostró autosuficiencia parcial 53\%, dependencia parcial 40\%, dependencia total 7\%. La categorización por riesgo demostró bajo riesgo $72 \%$, mediano $26 \%$ y alto $2 \%$. La complejidad de las atenciones relacionadas con actividades de autocuidado e intervenciones demandadas por su estado de salud determinó complejidad baja 79\%, mediana $18 \%$ y alta $2 \%$.

Se analizaron las fichas de eventos de los pacientes ingresados durante el año 2006 y 2007 que en agosto de 2008 llevaran un año en el Programa, lo que corresponde a 159 usuarios, detectando 124 eventos. El 44\% de los eventos fue de mediana complejidad (código amarillo del plan de contingencia), 32\% de alta complejidad (código rojo) y $24 \%$ de menor complejidad (código verde). Del total de eventos reportados $57 \%$ recibieron tratamiento médico en domicilio, 36\% requirieron hospitalización y el resto fue manejado telefónicamente por kinesiólogo, enfermera o a través de la línea telefónica de Salud Responde. La principal causa de alteración del basal clínico de los pacientes fue respiratoria, $73 \%$ del total de eventos. Sólo 2,4\% correspondió a falla de equipos.

Comparado con el histórico de los 12 meses previos al ingreso, hubo disminución de las hospitalizaciones durante el primer año de seguimiento, de $60 \%$ a $21 \%(\mathrm{p}=0,00001)$. Tam- bién hubo una disminución en el promedio de hospitalizaciones por niño y año (1,2 veces a 0,3 veces, $p=0,00001$ ).

La figura 3 grafica la evaluación de CVRS obtenida en 82 niños de la Región Metropolitana y sus familias comparando el momento del ingreso al programa con la evaluación a los 12 meses de su inclusión. La percepción de bienestar en salud aumento significativamente con los 2 instrumentos de CVRS, uno general y el otro específico para insuficiencia respiratoria: AUQUEI 17,2 \pm 5,6 puntos (56,3 \pm 7 vs 73,5 $\pm 4,4, \mathrm{p}<0,05)$, IRS 26,4 $\pm 8,2$ puntos (63 $\pm 10,1$ vs 89,4 $\pm 7,5$, p < 0,05). La escala IFS aumento 5,1 \pm 1,7 puntos (49,9 \pm 2 vs $55 \pm 1,3, \mathrm{p}>0,05$ ), destacando que en la dimensión de dominio (capacidad de la familia para abstraerse de la enfermedad y planificar actividades de recreación) se presento un aumento significativo (4,56 $\pm 1,2$ vs $7 \pm 0,6$, $\mathrm{p}<0,05)$. En la evaluación del Apgar familiar se observó un aumento de familias funcionales (59,5\% vs 66\%, p > 0,05). El Cuestionario de Goldberg presentó una disminución de los signos de trastornos del ánimo y alteraciones en el estado de ansiedad ( $37 \%$ vs 32\%, p > 0,05).

La correlación entre Pimax y espirometría se obtuvo al ingreso al programa en 26 niños con ENM. La mediana de edad fue 14 años (rango 8 a 18 años). La Pimax promedio fue 60,7\%. CVF 46,7\%; $\mathrm{VEF}_{1} 65,7 \%$ y $\mathrm{VEF}_{1} / \mathrm{CVF}$ $95,4 \%$. Se estableció una correlación modera-

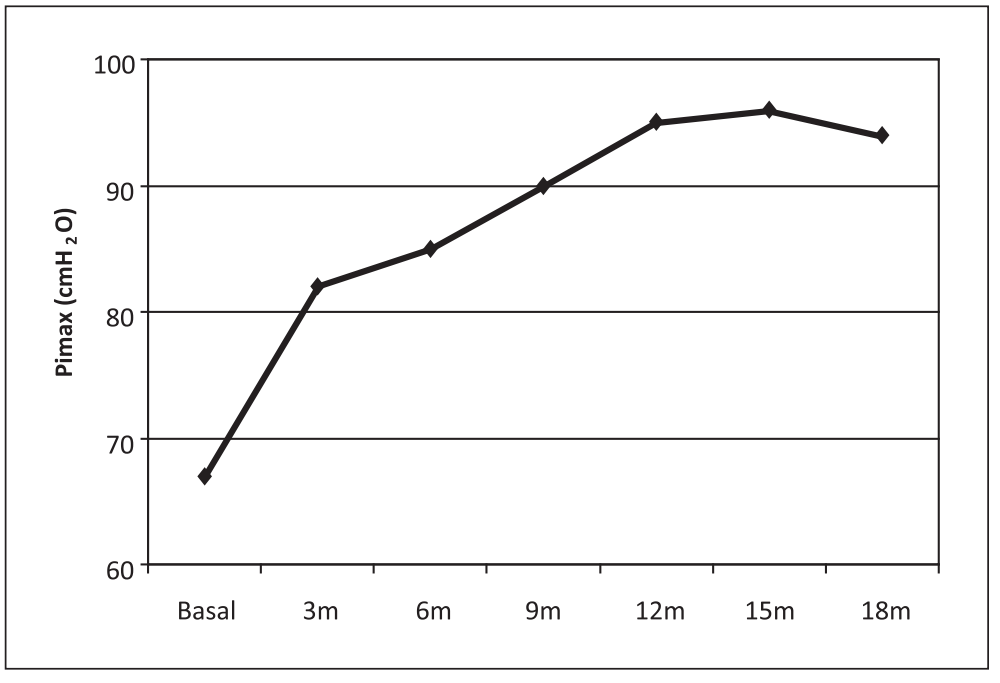

Figura 2. Aumento (\%) de la presión inspiratoria como porcentaje del límite inferior en 18 meses de entrenamiento muscular respiratorio. Pimax: presión inspiratoria máxima; $\mathrm{cmH}_{2} \mathrm{O}$ : centímetros de agua. 


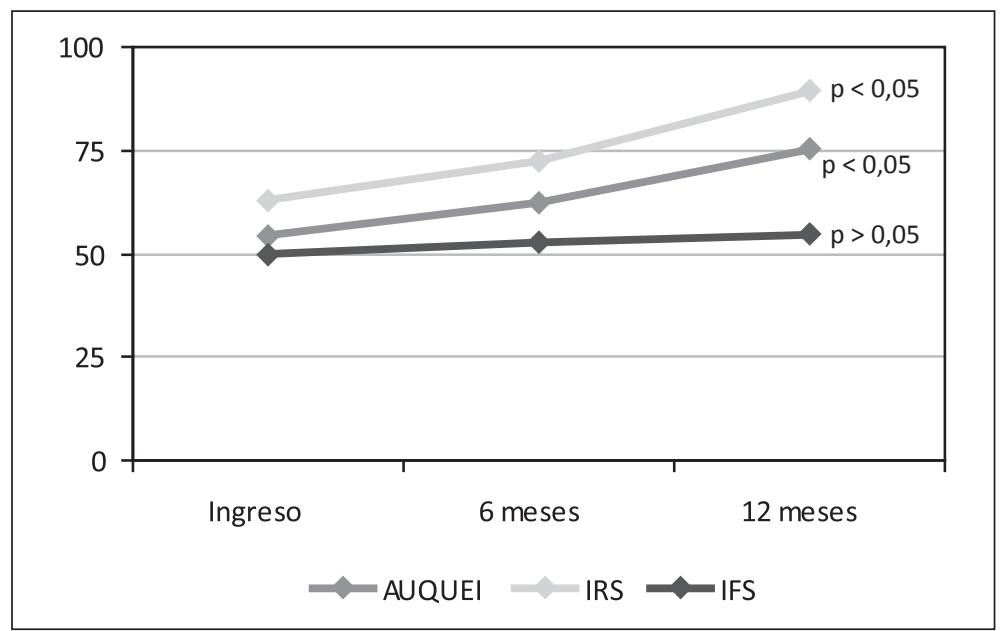

Figura 3. Calidad de vida relacionada con salud al ingreso, 6 y 12 meses de prestaciones en el programa AVNI, según AUQUEI, IRS e IFS. AUQUEI: Cuestionario autoaplicado de calidad de vida para niños con imágenes (Autoquestionnaire Qualité de VieEnfant-Imagé). IRS: Cuestionario de insuficiencia respiratoria severa. IFS: Escala de impacto familiar. Prueba comparación de medias de variables $\mathrm{T}$ de Student. da entre Pimax con CVF (Rho = 0,53; $\mathrm{p}=0,01)$ y Pimax con $\mathrm{VEF}_{1}(\mathrm{Rho}=0,49 ; \mathrm{p}=0,05)$. No se encontró correlación con $\mathrm{VEF}_{1} / \mathrm{CVF}$.

Se analizó el efecto del EMR en 21 niños con ENM que alcanzaron 18 meses de entrenamiento regular, la mediana de edad fue de 13 años (rango 8 a 16 años). Usuarios de BiPAP 14/21 (66\%). En ellos la Pimáx inicial fue 67 $\pm 22 \mathrm{cmH}_{2} \mathrm{O}$ y a los dieciocho meses fue de 94 $\pm 24 \mathrm{cmH}_{2} \mathrm{O}(\mathrm{p}<0,05)$. El delta promedio de Pimáx fue $27 \mathrm{cmH}_{2} \mathrm{O}$ (figura 2). La CVF fue de $61,2 \pm 26,6 \%$ al inicio y $63,2 \pm 31,7 \%$ a los 12 meses ( $<<0,1940)$.

\section{Discusión}

Pese a existir reportes de un programa similar en Sudamérica ${ }^{9}$, es el programa chileno de AVNI en domicilio, el primero en tener cobertura nacional y continuidad presupuestaria incorporando a beneficiarios del sistema público menores de 20 años ${ }^{16}$.

Existe escasa experiencia que analice la eficacia de la AVNI domiciliaria en pediatría entregada a través de programas específicos. Esta corresponde a encuestas o pequeñas series clínicas en niños y adolescentes con ENM, enfermedad del parénquima pulmonar con insuficiencia respiratoria crónica hipercapnica (fibrosis quística) y enfermedad de la vía aérea $^{1,2,4,5,7,8,10,24}$. Las indicaciones de AVNI que se utilizaron en este programa son similares a las descritas en la literatura internacional, manteniendo la heterogeneidad diagnóstica y los diferentes tipos de compromiso de la bomba respiratoria ${ }^{4,26}$. Es así, como los motivos para el inicio de la AVNI fueron similares a lo clásicamente reportado, insuficiencia respiratoria crónica hipercapnica, hipoventilación nocturna en pacientes con falla primaria de la bomba respiratoria, obstrucción de la vía aérea superior por malformaciones craneofaciales y apnea obstructiva del sueño. En nuestra serie la indicación siempre fue electiva y protocolizada incluyendo preferentemente elementos clínicos, alteración de los gases sanguíneos, desaturación en registros nocturnos extendidos y función pulmonar con restricción pulmonar severa o disminución de la fuerza muscular inspiratoria.

La AVNI utilizada con equipos de presión control bi-nivelada, mascarillas generalmente nasales y ciclados en forma espontánea, es una modalidad de uso habitual reportada en pediatría independiente del diagnóstico primario y que se relaciona con disminución de la carga del trabajo respiratorio, aumento de la ventilación minuto y aumento del intercambio gaseoso $^{10,26,27}$.

En nuestra serie, al igual que en otras publicaciones destinadas a evaluar la eficacia de la AVNI en exacerbaciones respiratorias, hubo hallazgos similares en la disminución de las hospitalizaciones ${ }^{6}$. Pese a que no hicimos una evaluación de costos, los costos directos mensuales de un paciente en su domicilio son, para 
el programa evaluado, al menos un tercio de los costos de una hospitalización prolongada. Aún cuando la mayoría de la morbilidad registrada fue de causa respiratoria y condicionó eventos de complejidad mediana o alta, los planes de contingencia permitieron su apropiada resolución en la red asistencial involucrando escalonadamente los escenarios del domicilio, atención primaria, sistemas de pre-emergencia, centros secundarios y terciarios. Comparado con el histórico la importante disminución de las hospitalizaciones, probablemente podría significar ahorro en los gastos en salud.

Los hallazgos descritos para la CUDYR, justifica que las prestaciones de los profesionales en el domicilio se basen en la supervisión y educación de acciones de autocuidado, permitiendo optimizar los recursos, logrando satisfacer proporcionalmente las demandas individuales y grupales ${ }^{17}$. Es interesante que a diferencia de lo reportado en otras publicaciones y en pacientes con ENM, nuestra evaluación a 12 meses de seguimiento en la CVRS y la evaluación funcional de la fuerza de la musculatura inspiratoria mostraran mejoría ${ }^{28,29}$. Es posible que los logros obtenidos en la percepción de bienestar en salud se deba a una metodología general y específica que evalúa las distintas dimensiones de la CVRS que podrían estar afectando al paciente desde un punto de vista integral. En este sentido, su aplicación y análisis permite identificar claramente que dimensión (relacionada con los síntomas, funcional o psicosocial) es necesario abordar, logrando establecer una intervención holística e interdisciplinaria, con intervenciones planificadas ${ }^{23}$.

La mejoría en la fuerza muscular obtenida en pacientes con ENM podría ser atribuida a un protocolo simultáneo de EMR. En el estudio de Mellies et $\mathrm{al}^{5} \mathrm{y}$ en nuestra serie hubo similitud en la estabilización de la CVF en pacientes con ENM con deterioro lento y progresivo. Estos resultados han sido descritos previamente por Koessler et al $^{29}$. Sin embargo, la repercusión de estos hallazgos, combinando AVNI y protocolos de entrenamientos específicos sobre los músculos respiratorios debe ser analizada en un mayor número de pacientes para tener conclusiones sólidas.
En este reporte la mortalidad fue baja y no se relacionó con complicaciones atribuibles a la AVNI. Las causas de muerte y la proporción fueron similares a una serie de 20 pacientes con VMP domiciliaria publicada recientemen$\mathrm{te}^{30}$. Es posible que la baja morbilidad y mortalidad observada se deba a los beneficios de la AVNI reportados en adolescentes y adultos con ENM y cifoescoliosis, categoría diagnostica mayoritaria de los pacientes incorporados al programa. En este grupo la AVNI prolonga la supervivencia en pacientes con hipercapnea ${ }^{6,7} \mathrm{y}$ en aquellos con normocapnia en vigilia (pero con hipoventilación nocturna) mejora la $\mathrm{SpO}_{2}$ y la $\mathrm{PaCO}_{2}$ durante el sueño, en relación a pacientes con las mismas características pero que no recibieron AVNI. De hecho estos últimos debieron ser incorporados a AVNI dentro del primer año de seguimiento por deterioro clínico o funcional diurno evidente ${ }^{2}$. Es probable que para los pacientes con enfermedad del parénquima pulmonar e insuficiencia respiratoria crónica hipercápnica, que en nuestro reporte fundamentalmente fueron niños con bronquiolitis obliterante post viral, la explicación sea similar, aún cuando los beneficios de la AVNI han sido reportados específicamente en pacientes con fibrosis quística, disminuyendo la declinación de la función pulmonar en etapas avanzadas de la enfermedad ${ }^{30}$, permitiendo ser un puente para el transplante pulmonar $^{3,31}$ y aumentando la sobrevida post transplante ${ }^{24}$.

Una limitación del presente estudio es el caracter retrospectivo, con heterogeneidad en los diagnósticos y en el tipo de compromiso de la bomba respiratoria y la ausencia de un grupo control, lo que limita las posibilidades de obtener conclusiones robustas de la efectividad de la AVNI domiciliaria y protocolos de EMR simultáneos. Por otra parte, existían datos incompletos de las fichas clínicas, por lo que no se obtuvo valores en la totalidad de los pacientes de la función pulmonar y presión inspiratoria máxima, lo que sumado a que un tercio de los pacientes son menores de 6 años, limitó las posibilidades de presentar estas características. En el caso de los pacientes con ENM con deterioro de sus habilidades neurocognitivas y/o otras condiciones clínicas, como compromiso 
de los músculos faciales, limitaron su colaboración para estos exámenes funcionales.

La evaluación de la CVRS y CUDYR no esta referido al total de la población, ya que se consideró a pacientes con seguimiento en centros urbanos para homogenizar los grupos y evitar variabilidad de los evaluadores.

La evaluación de ingreso y/o seguimiento objetivo de la hipoventilación nocturna con estudios de sueño y capnografía no estuvo presente en todos los pacientes debido al acceso limitado de estos en los centros hospitalarios del país y a que en un grupo de pacientes el deterioro clínico y funcional era evidente, asociado a que previo al inicio de las prestaciones del Programa no existían instancias formales que brindaran asistencia ventilatoria a estos pacientes, impidiendo hacer un reporte de estos datos en la serie reportada.

\section{Conclusiones}

Estos hallazgos sugieren que un Programa de AVNI es factible de ser implementado en niños y adolescentes, con criterios estrictos de selección, manejo interdisciplinario y acciones otorgadas por la atención primaria de salud en el domicilio en países en vías de desarrollo. En nuestra serie y con más de un año de seguimiento, esta estrategia permitió disminuir las hospitalizaciones, coordinar la red asistencial para la resolución de las exacerbaciones, mejorar la CVRS y posiblemente impactó en la mejoría observada en la fuerza muscular inspiratoria como en la ausencia de declinación en la función pulmonar en un grupo de pacientes con ENM.

\section{Agradecimientos}

A todos los profesionales y técnicos del Programa AVNI, Salud Responde y Servicio de Atención Médica de Urgencia por su labor incondicional con los pacientes en pos de lograr su mejor manejo y calidad de vida.

\section{Referencias}

1.- Fauroux B, Boffa C, Desguerre I, Estournet B, Trang $H$ : Long-term noninvasive mechanical ventilation for children at home: a national survey. Pediatr Pulmonol 2003; 35: 119-25.

2.- Ward S, Chatwin M, Heather S, Simonds A: Randomised controlled of non-invasive ventilation for nocturnal hypoventilation in neuromuscular and chest wall disease patients with daytime normocapnia. Thorax 2005; 60: 1019-24.

3.- Fauroux B, Le Roux E, Ravilly S, Bellis G, Clément $A$ : Long-term noninvasive ventilation in patients with cystic fibrosis. Respiration 2008; 76: 168-74.

4.- Consensus Conference: Clinical indications for noninvasive positive pressure ventilation in chronic respiratory failure due to restrictive lung disease, COPD, and nocturnal hypoventilation. A consensus conference report. Chest 1999; 116: 521-4.

5.- Mellies U, Ragette R, Dohna-Schwake C, Boehm H, Voit T, Teschler H: Long-term Noninvasive Ventilation in Children and Adolescents with Neuromuscular Disorders. Eur Respir J. 2003; 22: 631-6.

6.- Donha-Schwake C, Podlewski P, Voit T, Mellies U: NonInvasive ventilation reduces respiratory tract infections in children with neuromuscular disorders. Pediatric Pulmonologyc 2008; 43: 67-71.

7.- $\quad$ Young HK, Lowe A, Fitzgerald DA, Seton C, Waters KA, Kenny $E$, et al: Outcome of noninvasive ventilation in children with neuromuscular disease. Neurology 2007; 68: 198-201.

8.- Benditt J: Initiating Noninvasive Management of Respiratory Insufficiency in Neuromuscular Disease. Pediatrics 2009; 123: S236-8.

9.- Resener TD, Martínez FE, Reiter K, Nicolai T: Home ventilation of pediatric patients - description of a program. J Pediatr (Río J) 2001; 77: 84-8.

10.- Fauroux B, Lofaso F: Non-invasive mechanical ventilation: when to start for what benefits? Thorax 2005; 60 : 979-80.

11.- Simonds A: Recent Advances in Respiratory Care for Neuromuscular Disease. Chest 2006; 130: 1879-86.

12.- Simonds A: Respiratory support for the severely handicapped child with neuromuscular disease: ethics and practicality. Semin Respir Crit Care Med 2007; 28: 342-54.

13.- Sánchez I, Valenzuela A, Bertrand P, et al: Apoyo ventilatorio domiciliario en niños con insuficiencia respiratoria crónica. Experiencia clínica. Rev Chil Pediatr 2002; 73: 51-5.

14.- Bertrand P, Fielhmann E, Lizama M, Holmgren N, Silva M, Sánchez I: Asistencia ventilatoria domiciliaria en niños chilenos: 12 años de experiencia. Arch Bronconeumol 2006; 42: 165-70. 
15.- Prado F, Boza ML, Koppmann A: Asistencia ventilatoria no invasiva domiciliaria nocturna en Pediatría. Rev Chil Enf Respir 2003; 19: 146-54.

16.- Ministerio de Salud, Subsecretaria de Redes Asistenciales: Resolución Exenta $N^{\circ} 372$ del 29 de febrero de 2008: Programa de Asistencia Ventilatoria No Invasiva en Atención Primaria de Salud y Protocolo de Ventilación Mecánica Invasiva en APS.

17.- García MA, Castillo L: Categorización de usuarios: una herramienta para evaluar las cargas de trabajo de enfermería. Rev Med Chile 2000; 128: 177-83.

18.- Zenteno D, Puppo H, Vera R, et al: Guías de rehabilitación para niños con enfermedades respiratorias crónicas. Neumol Pediatr 2008; 3 (Supl): 25-33.

19.- Black LF, Hyatt RE: Maximal respiratory pressures: normal values and relationship to age and sex. Am Rev Respir Dis 1969; 99: 696-702.

20.- American Thoracic Society: Standardization of Spirometry, 1994 Update. Am J Respir Crit Care Med, 1995; 152: 1107-36.

21.- Szeinberg A, Marcotte JE, Roizin H, et al: Normal values of maximal inspiratory and expiratory pressures with a portable apparatus in children, adolescents, and young adults. Pediatr Pulmonol 1987; 3: 255-8.

22.- Knudson RJ, Lebowitz MD, Holberg CJ, Burrows B: Changes in the normal maximal expiratory flow-volume curve with growth and aging. Am Rev Respir Dis 1983; 127: 725-34.

23.- Salinas P, Farías A, González X, Rodríguez C: Calidad de vida relacionada en salud: Concepto y evaluación en pacientes con ventilación mecánica no invasiva. Neumol Pediatr 2008; 3 (Supl): 34-9.

24.- Efrati $O$, Kremer MR, Barak A, et al: Improved survival following lung transplantation with long-term use of bilevel positive pressure ventilation in cystic fibrosis. Lung 2007; 185: 73-9.

25.- Gozal D: Pulmonary manifestations of neuromuscular disease with special reference to Duchenne muscular dystrophy and spinal muscular atrophy. Pediatr Pulmonol 2000; 29: 141-50.

26.- Norregaard $O$ : Noninvasive ventilation in children. Eur Respir J 2002; 20: 1332-2.

27.- Prado F, Salinas P, Zenteno D, et al: Recomendaciones para los cuidados respiratorios del niño y adolescente con enfermedades neuromusculares. Neumol Pediatr 2010; 5: 74-88.

28.- Phillips MF, Quinlivan RC, Edwards RH, Calverley PM: Changes in spirometry over time as a prognostic marker in patients with Duchenne muscular dystrophy. Am J Respir Crit Care Med 2001; 164: 2191-4.

29.- Koessler W, Wanke T, Winker G, et al: 2 years' experience with inspiratory muscle training in patients with neuromuscular disorders. Chest 2001; 120:765-9.

30.- Ottonello G, FerrariI I, Pirroddi IM, et al: Home mechanical ventilation in children: retrospective survey of a pediatric population. Pediatr Int 2007; 49: 801-5.

31.- Caronia CG, Silver P, Nimkoff L, Gorvoy J, Quinn C, Sagy M: Use of bilevel positive airway pressure (BIPAP) in end-stage patients with cystic fibrosis awaiting lung transplantation. Clin Pediatr (Phila) 1998; 37: 555-9.

32.- Dayyat E, Kheirandish-Gozal L, Gozal D: Childhood Obstructive Sleep Apnea. Chest 2007; 2: 433-44.

33.- Zenteno D, Salinas P, Vera R, Brockmann P, Prado F: Enfoque Pediátrico para el Estudio de los Trastornos Respiratorios del Sueño. Rev Chil Pediatr 2010; 81: 445-55. 